\title{
MATHEMATICAL RECREATIONS.
}

Mathematical Recreations and Problems of Past and Present Times. By W. W. Rouse BaLL, Fellow and Lecturer of Trinity College, Cambridge ; and of the Inner Temple, Barrister-at-Law. London : Macmillan and Co., 1892. 8vo, pp. xii. +240 .

Perhaps "mathematical recreations" can be more or less sharply classified under two heads :

1. Particular problems treated by general mathematical theories and methods, but so that the layman may not recognize the general in the particular; as with those puzzles which really turn upon the solution of simple equations.

2. Theories or parts of theories, small and distinct enough for popular presentation, yet mathematical in the sense that, as with the Chinese rings or the tower of Hanoï, they deal with number, form and relation by consecutive reasoning whose methods, as far as independent of subject-matter, are essentially those of mathematics; perhaps mathematical too in the sense that, as with some topological studies useful toward function-theory, they are already auxiliary to larger doctrines recognized as main parts of the science, or that, as with some apparently disconnected properties of numbers, they bid fair to be included later in general theories.

Such "recreations," whether relating to pure or to applied mathematics, would naturally, as compared with most other mathematical studies, be more concrete, and would appeal rather less formally to the logical sense, and relatively more to imagination and ingenuity. But we must not forget Kirkman's caution, uttered in connection with his schoolgirl problem : "A solution .... to be mathematical and not tentative, must inform us whether or no, and why, [so and so] will .... be suitable; and [this] before trial and examination of the [particular solution]"-or at least, independently of such trial. It is commonly all-important "to see the secret of the symmetry." *

These studies may be of more value than we realize : not only as pleasant introductions to existing theories, but yet more as leading the amateur by short paths to new fields where, not merely as a gleaner, he may experience the satisfaction of adding to our present knowledge something which is of interest in itself, is largely mathematical in spirit and method, and may some day conduce to the great generalizations of mathematics proper. As the world's leisure increases, a certain democracy in science may be essential to the right

* Cambridge and Dublin Math. Journal, vol. 5, 260. 
mental and moral development of the race; a democracy which the rapidly increasing sweep, abstractness and subtilty of the older sciences might hinder were not fresh shoots continually thrown out nearer to the ground in the form of "recreations."

In the attractive little book before us, the phrase "Mathematical Recreations " appears to be used, consistently, nearly as above ; all the problems having a mathematical bearing. The phrase formerly included scraps of almost everything; much as, etymologically, "Mathematics" is synonymous with "Science." Part of the title pages of Leake's edition of Van Etten (London, 1674) runs thus: "Mathematical Recreations : or, a Collection of Sundrie excellent Problems, out of the Ancient and Modern Philosophers; as, Secrets and Experiments in Arithmetick, Geometry, Cosmography, Horologiography, ... Musick, ... . Chymistry, Water-works, Fire-works, \&c., not vulgarly manifest till now; "-and among the "mathematical" secrets here revealed are "How to charge a cannon without Powder," "How to make an infallible perpetual Motion," "How to practise excellently the reanimation of Simples," "How to make a Cone or Pyramidal Body move upon a Table" [by putting a live beetle under it !], "How out of a quantity of Wine to extract that which is most windy and evil, that it hurt not a sick person", and other recipes for domestic and magic art.

The present work is, in part, a brief summary by sample of what is mathematical and best in the three traditions of Bachet (1612, 1624), Leurechon-Mydorge (1630) and Van Etten-Oughtred (1653) : a tradition continued by Ozanam, Montucla, and Hutton (1803, 1840). But this is supplemented by abundant material taken from the classical writers, from Fermat down, and from articles in scientific jour. nals, especially of the past fifteen years. Good use is also made of the author's private note-book, and of Lucas's elaborate Récréations Mathématiques (1882-3). The work is a very judicious and suggestive compilation, not meant mainly for mathematicians, yet made doubly valuable to them by copious references. The style, in the main, is so compact and clear that what is central in a long argument or process is admirably presented in a few words: yet there is occasional ambiguity or inaccuracy, due now and then perhaps to fundamental misconceptions, but oftener to a kind of carelessness which one feels all the more constrained to notice, even at the risk of appearing hypercritical, just because it is not uncommon with otherwise good writers.

One great merit of this or any other really good book on such a subject is its suggestiveness : in running through its pages, one is pretty sure to think of additional problems on 
the same general lines. I will indicate a few such as we go on, in the hope that some reader may be tempted to work them up.

In the first two chapters are various elementary tricks, problems, paradoxes, games and puzzles; of arithmetic, geometry and topology. The specimen methods of finding a number thought of, are of course very simple : mere solution of linear equations, or application of elementary theory as to integers and factors or as to the Arabic notation; yet even here an instructive problem suggests itself to the reader, viz. : How to classify all such methods. So with the next trick, to find the result of a series of operations performed on an unknown number, i.e., so to arrange the operations that the number disappears from the result; and so when two or more numbers unknown to the conjurer have been thought of, or when an unknown selection or arrangement has been made from numbers known to him. Hints are given as to how these tricks may be made more mysterious, and may be connected with numbered things like dials, cards, etc. Then come, among other things, the counting-out problem ; the weights problem with Macmahon's generalizations; and a specimen of what we may describe as the puzzle of dividing $a+b+\ldots$. ounces of balsam into portions of $a, b, \ldots$. . ounces, when one has only $k$ jars, holding $m_{1}, m_{2}, . . . m_{k}$ ounces respectively. Chapter I. closes with an interesting account of some of Fermat's and Mersenne's speculations, especially as to the prime factors of $2^{m} \pm 1$. Among other good reasons given for suspecting that Fermat had some method, now lost, for finding the factors of large numbers, is this : that, in reply to a question from Mersenne as to whether 100895598169 had factors, Fermat wrote, apparently by the next day, * that they were the primes 112303 and 898423. And yet, from the prominence of numbers $2^{m} \pm 1$ in his own and Mersenne's researches, might not Fermat naturally suspect that the factors sought had some such form ; or at least, what is true, that one factor was nearly $2^{m}$ times the other? If so, he would probably think to divide the given number by 2,4 , 8 .. . , and to compare the quotient with the nearest perfect square : a procedure certain to have given the actual factors at the first trial.

Chapter II. begins with good samples of two kinds of geometric fallacy: what we may call the topological, where one hastily assumes (e.g.) that a certain intersection lies above and not below a certain line; and the cruder optical, where in the

* Fermat wrote: “Vous me démandez . . . une méthode pour découvrir, dans l'espace d'un jour, s'il est premier ou composé," etc. (Lucas, vol. 2, p. 230 ). 
absence of strict geometric analysis a slight inaccuracy of form goes undetected and the eye deceives us into thinking (e.g.) that a square $8 \times 8$ inches has been dissected into a rectangle $5 \times 13$. It might be of interest if some one would classify, exhaustively under his own chosen restrictions, all the great subdivisions of these and kindred geometric fallacies; and the like may be said of algebraic fallacies. Such classification would be the more useful because a resolved paradox is always instructive and may lead, by inversion or the reductio ad absurdum, to positive results, though perhaps only to very simple ones.

Then come the determination of the number of colors needed to keep all adjacent regions of a given map distinct, and the application of Euler's and like theorems to the physical configuration of a country. The known extension of these theories to the features upon a multiply connected closed surface is not given : e.g., upon a Riemann surface.

Of the games and puzzles of position that follow, some classes admit of further and perhaps indefinite development. About the best of these is concerned with arranging $m$ trees in $n$ rows of $p$ trees in a row, $m$ being as small, and $n, p$ as large as may be. Others are ferry-boat and shunting problems,- - the latter closely allied to the " 15 puzzle" which is given later, - and the study of tesselation, here barely mentioned but which might be made very interesting if connected with questions of symmetrical coloring in $m^{2}+n^{2}$, $2\left(m^{2}+m n+n^{2}\right)$, or $m^{2}+m n+n^{2}$ colors, according as the primary form used was the square, the equilateral triangle, or the hexagon.

Reiss's theory of the solitaire-board $*$ is referred to, and its leading idea might well have been given, for it is pretty and not difficult; moreover, this might suggest a like theory for boards with rows intersecting at $60^{\circ}$.

In subsequent chapters are treated problems connected with cards, the "fifteen puzzle," the tower of Hanoï, the Chinese rings, the $n$ queens problem, Kirkman's arrangements of school-girls, magic squares; also unicursal problems, including those of mazes, of Hamilton's "game," and of the knight's path. Most of these ingenious puzzles have instructive theories here admirably stated, or are valuable as studies of symmetry.

Of the $n$ queens problem,-to place $n$ chess-queens on a board $n$ cells square so that no queen can take another,-all the "fundamental solutions" are given up to $n=8$ inclusive. The statement (p. 88) that, whenever $n=4 m+2$, a solution $246 \ldots n 135 \ldots(n-1)$ cxists $-i$. e.,

* Crelle's Journal, vol. 54, 344, or Lucas, vol. 1, 89. 
one wherein the columns $1,2,3, \ldots$ correspond respectively to the rows $2,4,6, \ldots$. - is not true when $m$ has the factor 3 . Two variations of this problem will suggest themselves: the tangents of the direction-angles of the queen's lines of action, viz. $0, \infty, \pm 1$, may be replaced by $\pm \frac{1}{2}, \pm 2$ as in the knight's move, or by other simple ratios of integers; and secondly, whether the directions be thus replaced or not, the board may be made self-returning vertically and horizontally, so that next after row or column $n$ comes 1 again, - a change that allies the problem to the elementary theory of numbers.

With the usual queen's move, every solution for a horizontally self-returning board will hold for a board that is also vertically self-returning; and every such solution will give another, or be reproduced, not only when the board is revolved or reversed, but when we cyclically permute either rows or columns in the order $123 \ldots n$, and likewise when we cyclically permute rows and columns alike in the order 1 , $1+r, 1+2 r, \ldots$ wherein $r$ is prime to $n$. I find that, for $n<12$, all such solutions are in these ways reducible to three fundamental ones: $13524,135 \% 246,135 \% 911246810$, the last of which becomes $14 \% 1025811369$ when the cyclic permutation $147 \ldots$ is applied to both rows and columns. Solutions, having like these four the forms $135 \ldots$ and 147 ...., exist whenever $n$ is prime to 6 ; but the question remains whether, for $n>11$, solutions not of the form $1,1+s$, $1+2 s, \ldots$ also exist.

Some of the school-girl and unicursal problems have a general mathematical interest; and the clear and rather full treatment of them will abundantly repay perusal, and leaves apparently little to criticise. The same is true of Magic Squares, chapter V.; though perhaps a little more might well have been said of Nasik squares and cubes, and of the property that the sum for every four adjacent cells of the self-returning board shall be $2 n^{2}+2$. How far can corresponding properties be secured on self-returning boards when the sub-squares considered have (say) 9 cells each instead of $4, n$ being odd, or when instead of rows, columns and diagonals, we consider lines parallel, for instance, to a chess-knight's moves? Among the few corrigenda to be made, is this: p. 116, 1. 22, and p. $11 \%$, 1. 9, read " . . in any order whatever that puts complementary numbers in complementary positions."

Chapter III., on "Some Mechanical Questions," is by far the most open to criticism, and must have been written or compiled in haste; but the "questions" themselves are instructive, and so are some of the oversights.

After an excellent refutation of the paradox of Achilles and the tortoise, and a rather less happy reply to the paradox 
that nothing can move, since nothing can move where it is nor yet where it is not, we are shown how an ice-boat, gliding without friction or lee-way, may sail faster than the wind. The formula given for the boat's velocity is right, and is deducible from the condition that the wind's motion relative to the sail be along the sail; but the pressure is wrongly taken to vary generally as the normal component of the wind's motion relative to the sail. Probably for a very broad low sail, the pressure varies nearly as the square of that component; while, for a very narrow high sail, Rayleigh's formula (Phil. Mag., Dec. 1876) would give

$$
p=0.005 v^{\prime 2}(4+\pi) \sin \varphi /(4+\pi \sin \varphi),
$$

wherein $v^{\prime}$ is the wind's relative velocity in miles per hour, $\phi$ the angle its direction makes with the sail, and $p$ the normal pressure in pounds per square foot; and for a plane sail of ordinary dimensions, the law would be intermediate in character.

Next is discussed a curious kind of boat-racing where the crew simply jerk a rope tied to the after thwart. "The effect of each jerk is to compress the boat. Left to itself the boat tends to resume its original shape, but the resistance to the motion through the water of the stern is much greater than that of the bow and hence on the whole the motion is forwards." Doubtless the different shaping of bow and stern does help the effect; but any sensible distortion of the boat seems impossible, neither would it much help matters after all. But if, with each jerk, the crew's center of gravity moves toward the bow $m$ times more rapidly than it moves back on the return, then all is explained; for the boat (supposed for simplicity not to be already under headway) is thrown backward nearly $m$ times as ropidly as forward, causing a water-pressure at the stern at least $m^{2}$ times as great as was that at the bow, though lasting only $1 / m$ as long.

Then come philosophical remarks on force, inertia, centrifugal force, work and equilibrium; all good enough but for occasional bits of carelessness. Thus (p. 61) we are told that, in throwing an irregular body or in rifling a gun, one tries to give the projectile a rotation about a principal axis; but as no reference has been made to the action of the air, the reader is left to infer that the rotation somehow guides the projectile under the laws of inertia. Then, after an anecdote from which perhaps the author's political sympathies may be surmised, comes Montucla's estimate of the time Archimedes must take in order to "move the world" one inch : over $3 \times 10^{12}$ centuries. It would indeed take some such time to raise by one inch, against terrestrial gravity, a mass equal to 
the earth's ; but nothing has been said of gravitation, only of mass, inertia, etc., and using Montucla's data we know that to move the earth one inch, just as gravitation moves a falling body from rest, would have taken Archimedes only about six weeks.

Finally, after some good pages on gravitation-toys, perpetual motion, and the want of stiffness in large models as compared with small, we come to "Motion of Fluids and Motion in Fluids." Here the author, making use of Hanksbee's empirical law that "when a fluid is in rapid motion, the pressure is less than when it is at rest," forgets that the diminished pressure observed is not the effect but only the cause of the more rapid motion. The fluid is accelerated in flowing from a region of greater pressure to one of less ; but a forced acceleration by bellows or blast-fan may not diminish the pressure. Were it right to reason as if mere concomitance among phenomena were causation, we should often find the Hauksbee effect offset, or more, by transmitted pressure from the fan, etc. Thus it is not true (p. 71) that, when we blow through a tube made by placing a paper on two books laid parallel, "the paper will be sucked in," unless the tube be larger than the entering current; nor that a "disk will be drawn towards" a simple cylindrical tube that blows squarely upon it. If the tube flare, or have something like another disk at its mouth, the effect named comes from a partial vacuum between the flaring sides or the two disks. In the pretty experiment of blowing "close and parallel" to a card (p. 7\%), the card's motion toward the blast is due to the lateral inrush of air to replace what has mingled and been carried off with the blast.

What suggested the law to Hauksbee (see Phil. Trans. 1704, p. 147) was the low barometer during a certain hurricane; and he adds the shrewd remark, which with our better means of observation might be followed up, that "on extraordinary gusts a visible vibration of the quicksilver appeared." [Does the "vibration" precede or follow the gust, and does it begin with a rise or a fall ?] - Yet even here we see how unsafe a reed, to lean upon, this law would be ; for though on the whole the barometer does commonly fall as the wind for a while rises, while the storm-centre is approaching, yet at the very centre the lowest pressure of all is most apt to accompany a brief calm.

To explain the deviation of a curved ball in the air on the principle that where the motion is greatest the pressure must be least, the author has to assume that the path curves toward that side of the ball whose motion is forward by virtue of rotation as well as of translation; but tennis-players tell us the reverse, and from the following experiment it seems that they 
are right. Hang a tennis-ball by a doubled string from the ceiling, twist the string, and swing the pendulum. If the string, in untwisting, spins the ball counter-clockwise, then the plane of oscillation slowly shifts counter-clockwise, showing that the right-hand side of the ball's path is convex; and when, presently, the ball begins to revolve the other way, so does the plane of oscillation. The result is the same, if a vertical wall be parallel and close to the plane of vibration. I do not know how completely the true theory of a curved ball has been worked out. It is said, and I suppose rightly, that when the ball, spinning counter-clockwise, flies northward, the friction heaps up a mass of air N.E. of it which travels with it and takes largely the brunt of the resistance to the ball's flight, and whose base giving way to the S.E. and S.W. pushes the ball to the west. Further, I conceive that the atmosphere carried along by the ball and spinning with it is gradually changed by diffusion and by the wind of translation, being mainly collected on the north side and blown off on the south side, carrying with it its eastward motion while the ball recoils to the west. The two actions are somewhat connected; but were the ball replaced by a long broad band between two small pulleys, the first action would be the chief if, in their flight, the pulleys followed one another, and possibly the second if they moved abreast.

It is stated (p. "71) that, "in a perfect elastic fluid . . . $p=\Pi \alpha^{-v^{2}}$ " where $p, v, \Pi, \alpha$ denote the pressure, the velocity, and constants. This is true only if $\gamma$, the ratio of the specific heat under constant pressure to that under constant volume, be 1 ; but for every known gas $\gamma$ exceeds 1 , and it must do so theoretically ; indeed, for what is called sometimes the perfect gas of theory, $\gamma=1.6^{6 \%}$, while for the more perfect of actual gases, as hydrogen, $\gamma=1.42$.

The chapter closes with the " unexplained" fact that some large birds follow a ship rapidly and long with no perceptible motion of the wings. It has been suggested, as a possibility, that they utilize air-eddies made by the ship, keeping in the up-draught. By Rayleigh's formula this might be, if the effective wing-surface were equivalent, by reason of its good shape, to 3 square feet of Rayleigh's resisting plane per pound of the bird's weight, and if the bird's horizontal resistance were negligible, his velocity relative to the wind 15 miles per hour, and the upward current 3 miles; but these assumptions may appear extravagant. It is known that, to a less extent, any differences of horizontal air-currents could also be utilized. I was once told by a seaman, I know not how accurately, that he had seen albatrosses (?) hover over the deck, apparently motionless against a stiff breeze, but really with a rapid wing-tremor of very small amplitude. This would help : 
the overlapping feathers would simply open as they rose, and close up as they descended.*

The last eighty pages, entitled "Mathematical Problems and Speculations," form almost a separate work. Beside brief and clear sketches of successive attempts to double the cube, trisect the angle and square the circle; of astrology; and of calendars and the ordinary measurement of time-they take up two topics of great philosophical interest : hyperspace, and the constitution of matter.

By "hyperspace" is meant not only the space of $n$ dimensions but also the so-called "curved space" of pan-geometry. The author holds that the hypothesis of our 4-dimensional existence helps to clear up some difficulties in the conception of the ether, in the explanation of gravity, in the supposed fact that the number of kinds of matter is finite and all atoms of a kind are alike, and in the approximate periodicity of atomic weights and other properties of the elements. To our space and to all bodies in the universe, he gives a uniform thickness in the fourth dimension, so that in that dimension they rest on, or in, a homogeneous thin elastic " medium," which is either a body or simply a hyperspace, and by whose vibrations all force and all radiant energy are transmitted. This medium must be thin in the fourth direction, since these energies are as the inverse square and not the inverse cube, etc., of the distance, unless the distance be very small; but its thickness has the same order of magnitude as atomic or molecular diameters, so that atomic forces do not follow the law of the inverse square. It secures that the number of modes of atomic vibration be finite, and hence also the number of kinds of matter,_- "as two clocks whose rates are nearly the same tend to go alike if their cases are connected." Finally, if the atoms were formed by some simple or complex harmonic disturbance, the formation might well occur only at a particular phase or phases; and atoms so formed might naturally run in series, as in Mendeléef's periodic law. At least, so runs the dream.

These suggestions appear to me the finest thing in the book. They are but little developed, and not always clearly: e.g., passages on page 197 may seem to imply, contrary to what we know of polarization, that the transverse luminiferous vibrations are in the fourth dimension. $\dagger$ Nevertheless the possibilities of the proposed theory may be immense. Of course all depends upon the success with which it can be applied not only to philosophical generalities, but to the definite predic-

* See Nature, vols. 8, 9, 23, 27-29, 35-37, 40, 42-44 ; and Science, vols. $10,11,13,17$.

$\uparrow$ See also Messenger of Mathematics, XxI., pp. 20-24. 
tion of laws ; still, a priori, the hypothesis would seem to be quite admissible. Certain details of the theory remain to be fixed as may be most advantageous. For instance, the medium may have either one or more dimensions besides the familiar three, and its extent in the new directions is as yet undetermined. It may be self-returning in the new directions, instead of ending abruptly; and so on. It may even shade off in the new directions, the materials of the world tending toward our three-fold space as a region of maximum density; either through some kind of selection such as Mr. C. S. Peirce has suggested, or of quasi-attraction, a little as in Hinton's Scientific Romances. Such shading would follow some rapid exponential law, as in the theory of distribution of errors; and its modulus, however small, would probably be definite.

The useful assumption "that the measure of distance remains the same everywhere" (p. 201), does not necessarily imply that every so-called "curved space" lies in an uncurved space of more dimensions, but only that the relation among the mutual distances of four quasi-complanar points be always that known to hold among the geodetic distances apart of four points on a sphere whose radius is either real or purely imaginary. Thus it is not true that a two-dimensioned pseudospherical space must be finite unless " constructed in space of four dimensions" (pp. 200-1) : and the oversight, made by so good a writer, goes to justify Klein's criticism that the phrase "curved space" is misleading. Why not rather, in the general case, describe the space as quasi-curved?

The rest of the account given of hyperspace and of the constitution of matter is well thought out and clear. It will help the general reader toward some truthful notions as to studies which may perhaps play an important part in the near future.

IтHACA, N. Y., November 15, 1892. J. E. OLIVER.

\section{RECENT STAR CATALOGUES.}

Zweites Münchener Sternverzeichniss, enthaltend die mittleren Oerter von 13200 Sternen, für das Aequinoctium, 1880. Beobachtet und berechnet von Dr. Julius BaUschiNGER, Observator der Sternwarte. Munich, 1891. 4to, pp. xxvi. and 172.

THE Scottish astronomer, John Lamont, long director of the observatory at Bogenhausen, a suburb of Munich, had caused the observation, about the middle of this century, of about 33,000 stars; they were taken in zones, and by an ingenious method for saving labor. Unfortunately, the assist- 